\title{
Outlooks on wearable technology products
}

\begin{abstract}
This review aims at giving a brief depiction of the current developing picture of wearable technology mainly from practical aspect, a straightforward and aculeate outlook for this primarily commercially driven and fast-changing field. We first discuss some general issues including the relationship with human physiology and psychology, the unique characteristics of wearable products and some technological obstacles hindering them from mass commercial production. We finally offer a nearfuture prediction based on our discussion, albeit concise.
\end{abstract}

Keywords: wearable technology, society, human body, commercial, prediction
Volume 3 Issue 3 - 2017

\author{
Manlin Song, Ning Pan \\ Division of Textiles and Clothing, Biological \& Agricultural \\ Engineering, The University of California at Davis
}

Correspondence: Ning Pan, Professor in Fiber \& Polymer Science, Division of Textiles and Clothing, Biological \& Agricultural Engineering, The University of California at Davis, USA,Tel 5307526232, Fax 5307527584,Email npan@ucdavis.edu

Received: October 29, 2017 | Published: November 24, 2017

\section{Introduction}

\section{Current developing status of wearable technology}

Wearable technology, also known as wearables, fashion electronics or smart clothing, refers to the techniques in developing novel functional products that can be incorporated into cloth worn on the body or even in the body as implants. ${ }^{1}$ The design principle builds on the interactions between user's body and the devices, as an extension of users' body and brain, and is hence entirely customer experience oriented.

Due to the special functions with intimacy to human body in sensing physiological signs, wearable devices are beyond the handheld electronic personal products such as cell phones even though they may frequently carry out the same tasks. ${ }^{2}$ One way to examine the products of wearable technology is from the customer experience, so that all the products can be categorized into two different sections: apparels and accessories. Accessories include wristbands, watches, shirts, shoes, shorts, caps, headbands, eyeglasses, belts and necklaces, etc., capable of responding to environmental stimuli and body biomedical signals, and are detachable from the cloth itself. Whereas the apparels are garments where such functions or devices have been incorporated to form coherent or more permanent clothing systems. The wearable devices mostly contain sensors to gather raw data and then connect to a database or software application for further analyzing and even responding. ${ }^{3}$

It is clear that wearable technology is a field that was largely initiated by industries and manufacturers focusing on the market share and profitability, using the science and knowledge already made available by academics in laboratories. Consequently, some of the more scholarly issues such as terminologies and manufacture processes are yet to be standardized: there are much less research papers in academic journals than the promoting pieces for specific product on popular press and websites. It seems many aspects about wearable technology are still fluidic. It would not be surprising that by the time this review article becomes public, some of the statements in it could appear obsolete already.

One of the present authors has already published a review article focusing more on the scientific and technical issues in wearable technology Pan \& Postle. ${ }^{4}$ What can be said so far is that there are three primary components or pillars supporting wearable technology: the textile substrates, microelectronics and computer control. The textile substrate serves as an indispensable buffer or interface between the wearer and the electronic devices. ${ }^{5}$ The microelectronics are the core component in rendering new functionalities to the cloth Pentland, ${ }^{6}$ including supplying sensors and powers to capture the bio-signals from the wearers; and the computer programs control and monitor the system operation, and process and interpret the data generated to notify the wearers when needed.

Many people think that wearable technologies depend solely on the future development of microelectronics and that the textile represents only an inert substrate that plays very little if any creative part. However, in order to achieve commercial success of truly integrated wearable technologies, we must develop the basic elements of the new technologies which are compatible with textile manufacturing practices, be it new battery technologies, textile based input and output systems, flexible sensors etc. To succeed, creative multidisciplinary research and coordinated manufacturing processes between very different industries are imperative.

\section{Current wearable technology applications}

As an emerging, cutting-edge technology field with a potentially huge and profitable market, the application of wearable technology is flourishing in different areas, especially in medical treatment, rehabilitation, military missions, professional sports, life quality and aesthetic alternation, etc., as roughly grouped; all requiring inter- and multi- disciplinary collaborations. These wearable applications have revitalized the research vigor in garments and accessories development with new functional, social, industrial, educational significances. Wearable technology is even considered "the key to the optimal operation of future society", ${ }^{7}$ especially in the following four main application areas: healthcare, safety, lifestyle, and arts. For instance, wearables can provide an array of valuable functions, including a realtime monitoring and in-time communication between patients and doctors to lower the emergency risks; a better safety precaution for certain occupations such as soldiers, astronauts, firefighters, polices and miners and other vulnerable groups to reduce or even eliminate accidents; convenience in our daily life being informed about the body state and physiological signs, reminding or automatically handling of many routine chores; possibility of realizing artists' otherwise dreaming ideas, rendering novel artistic forms and styles, and exploring new trend in art circles. All of these promise a truly 
exhilarating future. Here are four examples from healthcare, safety, lifestyle and arts representatively to show the development status of wearable technology.

Wear Sens-ingestion regulator: Engineers and scientists at University of California, Los Angeles designed a necklace of an innovative nutrition monitoring system to monitor food and drink intake as shown in Figure 1. User's muscle motion will trigger the sensors and transmit the signals from necklace to a computer algorithm so that the data will be translated into such information as the kinds of food or drinks and the amount the user has taken. The app on a smart phone will instruct whether the wearer should eat or drink less or more by comparing with previous records. According to the experiment results, Wear Sens could correctly identify the actions such as drinking, swallowing, and chewing with a relatively high precision of 90.17 percent. This technology could be very helpful to diabetics, obesities and allergic groups to track and improve users' dietary habits. ${ }^{8}$

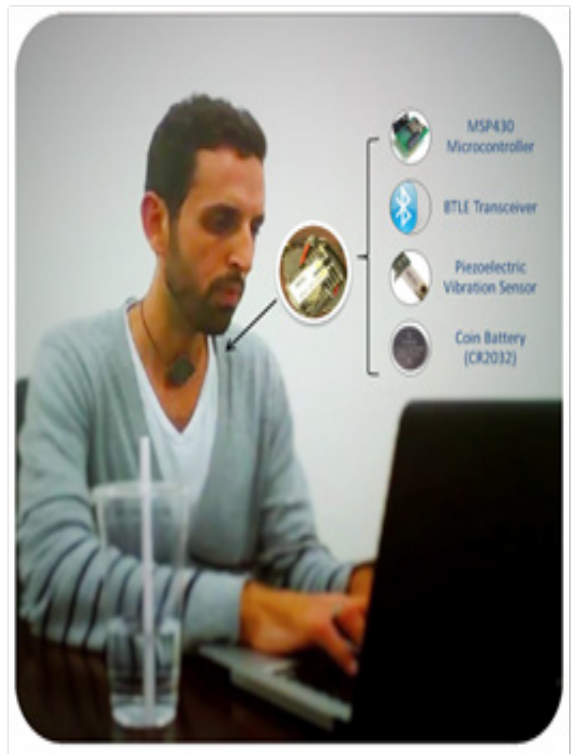

Figure I Wear Sens-an ingestion regulator Kalantarian et al. ${ }^{8}$

Save one life intelligent insole: Lemur Design Studio, a Colombian company, developed the Save One Life $^{9}$ intelligent insoles with military mine detector, to protect soldiers' lives. The smart insole set shown in Figure 2 comprises of a footpad using conductive materials with physiology-detective microprocessors and a wrist device to receive warnings from radio signal to display and locate the suspicious objects. Such metal detectors containing printed circuits installed in the sole will release a warning signal on the watch to alarm soldiers to dangers or to walk around the mines after the patrols have detected the risky areas. This technology effectively decreases the risk of mine triggering.

Aurora-powered suit: A startup and spinoff of SRI international called Super flex is working on powered, lightweight and connected clothing called "Aurora Powered Suit" 10 in Figure 3. The design of Aurora comes up with an idea to solve the mobility problem of the elders in such basic needs of getting up, sitting down or staying upright. Using the knowledge of biomimicry and ergonomics, this cloth helps the aging generation support the torso, hips, and legs while sitting, standing up and moving around. With the cooperation with Yves Behar and his design firm Fuse project, the functional garments are not only equipped with electric muscles, battery, sensors, motors and control panels, but also fashionable and comfortable. The company may release their commercial products by the middle of 2018 .

Data Dress: Google and HM Ivyrevel Perez ${ }^{11}$ create a personalized and customer-made dress in Figure 4 that can be ordered through a forthcoming application on smart phones. Snapshot API is used to monitor users' daily activities and lifestyles, mainly about the places they go and the weather in their area on the map. This information will be collected and translated to create digital pattern on the dress automatically by the app, and users can do various modifications like styles, colors, and patterns to further personalize the dress of their own aesthetic taste. Later on, the dress combining the life paths and personal preferences could be ordered online.

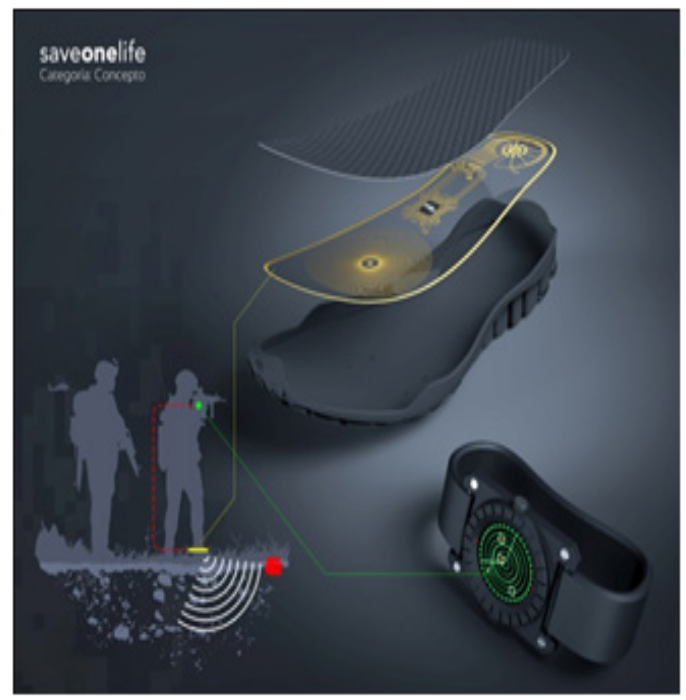

Figure 2 The Save One Life intelligent insole. ${ }^{9}$

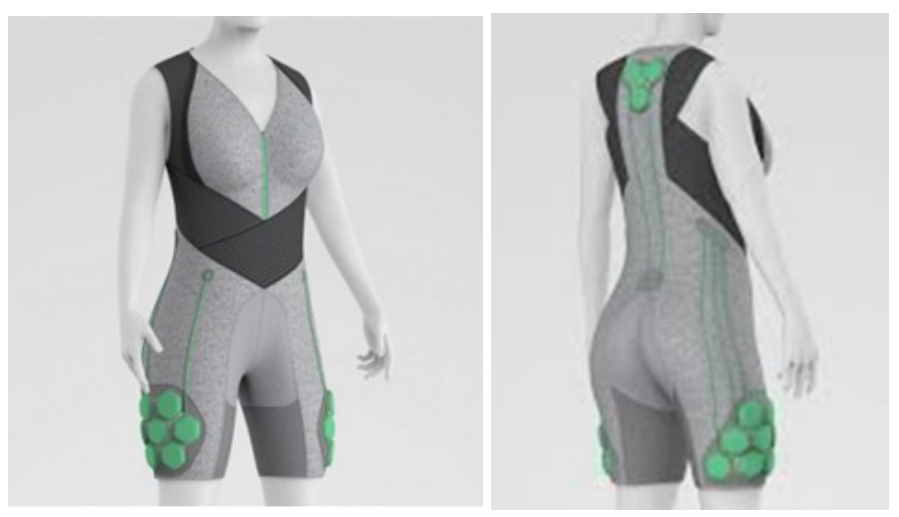

Figure 3 The Aurora powered suit.

\section{Future predictions on wearable technology products}

For the future, projections by different sources of wearable technology vary in magnitude but consistent in high growing potential. Besides the concerns discussed above that require attention and solutions, even for currently successful products, there are still ample rooms for further improvement, based on the continuous research, the changing social structures and assumptions about the body-cloth interactions.

Applications of wearables will expand to more areas and adapt to more social groups and cohorts. Besides its proving applications in 
medical areas for detection, treatment, prevention of several diseases Bonato ${ }^{12}$ Liang \& Yuan, ${ }^{13}$ there is emerging research working on mental diseases. For example, in order to facilitate the psychotherapy treatment such as cognitive behavioral therapy, the Aaron McEuen's group developed a device called "\&You" to detect measurable anxiety symptoms of the patients with stress disorders, and then with vibration sensors to generate stimuli to calm down the patients by adjusting their body position to expand their chest and lung capacity. ${ }^{14}$

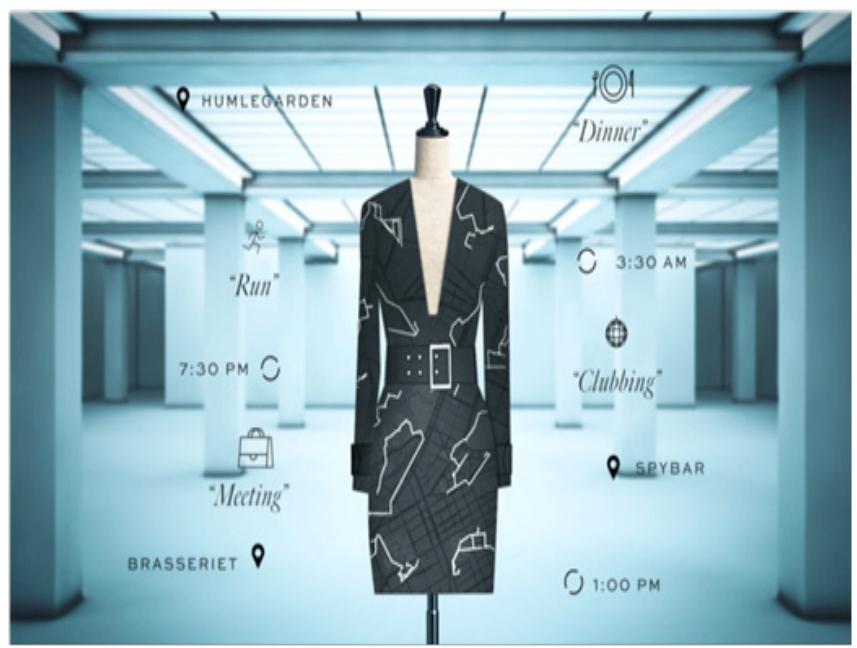

Figure 4 The data dress.

Design of wearables is expected to exert a near-revolutionary impact on the conventional design aesthetic criteria and breaking the new ground for fashion media. Wearables are supposed to be more body-friendly both in garments and accessories, more comfortable, and with more flexible and fabric-based sensors; accessories-based sensors are going to be smaller, easy-potable and even as indiscernible as nail polishes, buttons, earrings, stickers or hairs Pan \& Postle. ${ }^{4}$

Still a much closer collaboration among scientists, engineers, and designers is indispensable for the wearable industry to sustain Seymour. ${ }^{15}$ If design and technology are expected to fuse together seamlessly, both research institutes and industries of design and technology need compromise to speak common tongue and cooperate to create successful products (University College London, 2014). It is necessary to further explore the common ground that bonding individuals' performance practice with wearable interaction Schiphorst. ${ }^{16}$ Such interdisciplinary research will offer good educational opportunities as well to motivate more students with interest and abilities in research and teamwork in wearable areas.

Wearables enable a refresh and unprecedented thrust to enhance individuals' experience by having much more frequent interactions between human body, computers, and physical environment than before. These interactions are emerging and developing under particular cultural, historical and social background. ${ }^{17}$ It is essential to guarantee that these interactions are useful, pleasurable, and most importantly, meaningful. ${ }^{18}$ Internet networking and micro sensors have been permeating through everyone's life in a ubiquitous way and be a part of our life ultimately. Social influences will have a significant impact on acceptance and usage of brand-new technologies Malhotra $\&$ Galletta $^{19}$ and the acceptance of wearable technology will in turn bring in a new phrase in society. While young generation still are the main part in embracing this revolutionary innovation, social perceptions of wearable technology overall are increasingly evident and many studies have revealed a considerable social adoption by break various barriers; ${ }^{20,21}$ Profita et al. ${ }^{22}$ which will bring more research impetus to this field.

\section{Acknowledgements}

None.

\section{Conflict of interest}

Author declares there is no conflict of interest.

\section{References}

1. WT. Interview with Nitin Pai-SVP of Marketing and Strategy, Tata Elxsi. 2017

2. Wright R, Keith L. Wearable technology: If the tech fits, wear it. J Electronic Resources in Medical Libraries. 2014;11(4):204-216.

3. Park S, Jayaraman S. Enhancing the quality of life through wearable technology. IEEE Engineering in Medicine and Biology Magazine. 2003;22(3):41-48.

4. Pan N, Postle R. 10 Commandments" in Wearable Technologies. China Textile Leader. 2016;24:54-57.

5. Park S, Jayaraman S. Smart textiles: Wearable electronic systems. MRS bulletin. 2003;28(8):585-591.

6. Pentland AP. Wearable intelligence: Scientific American Incorporated. 1998. p. 90-95.

7. WT. Wearables in Healthcare. 2017.

8. Kalantarian H, Motamed B, Alshurafa N, et al. A wearable sensor system for medication adherence prediction. Artif Intell Med. 2016;69:43-52.

9. Techno Crazed. 2017.

10. Superflex. 2017

11. Perez S. Google \& H\&M's Ivyrevel will make you a dress customized using your personal data. 2017.

12. Bonato P. Wearable sensors and systems. From enabling technology to clinical applications. IEEE Engineering in medicine and biology magazine. 2010;29(3):25-36.

13. Liang T, Yuan YJ. Wearable Medical Monitoring Systems Based on Wireless Networks: A Review. IEEE Sensors J. 2016;16(23):8186-8199.

14. McEuen A, Proffitt J, Camba JD, et al. \&You: Design of a Sensor-Based Wearable Device for Use in Cognitive Behavioral Therapy. Advances in Human Factors and Ergonomics in Healthcare. 2017. p. 251-260.

15. Seymour S. Fashionable technology: The intersection of design, fashion, science, and technology. 2008

16. Schiphorst T. Breath, skin and clothing: Using wearable technologies as an interface into ourselves. International $J$ of Performance Arts and Digital Media. 2006;2(2):171-186.

17. Berzowska J. Memory rich clothing: second skins that communicate physical memory. Proceedings of the 5th conference on Creativity \& cognition. 2005. p. 32-40.

18. Berzowska J. Electronic textiles: Wearable computers, reactive fashion, and soft computation. Textile. 2005;3(1):58-75

19. Malhotra Y, Galletta DF. Extending the technology acceptance model to account for social influence: Theoretical bases and empirical validation. Systems sciences, HICSS-32. Proceedings of the 32nd annual Hawaii international conference on. 1999. 
20. Holleis P, Schmidt A, Paasovaara S, et al. Evaluating capacitive touch input on clothes. Proceedings of the 10th international conference on Human computer interaction with mobile devices and services. 2008. p. $81-90$.

21. Page T. Barriers to the Adoption of Wearable Technology. $i$-Manager's $J$ Information Technology. 2015;4(3):1.
22. Profita HP, Clawson J, Gilliland S, et al. Don't mind me touching my wrist: a case study of interacting with on-body technology in public. Proceedings of the 2013 International Symposium on Wearable Computers. 2013. p. 89-96. 